\title{
Spectroscopy and Photophysics of Self-Organized Zinc Porphyrin Nanolayers. 2. Transport Properties of Singlet Excitation
}

\author{
Harry Donker,* Arie van Hoek, Willem van Schaik, ${ }^{\dagger}$ Rob B. M. Koehorst, \\ Mikalai M. Yatskou, ${ }^{*}$ and Tjeerd J. Schaafsma \\ Laboratory for Biophysics, Department of Agrotechnology and Food Sciences, Wageningen University, \\ Dreijenlaan 3, 6703 HA Wageningen, The Netherlands
}

Received: February 16, 2005; In Final Form: May 19, 2005

\begin{abstract}
Exciton diffusion has been studied in 5-25-nm-thick films of zinc tetra-( $p$-octylphenyl)-porphyrin (ZnTOPP) spin-coated onto quartz slides by intentional doping with quenchers using steady-state as well as time-resolved fluorescence spectroscopy. The fluorescence spectra of the films are very similar to those of solutions, indicating emission from localized exciton states. From the dependence of the fluorescence quenching on the quencher concentration and fluorescence lifetime measurements, the exciton diffusion can be concluded to be quasione-dimensional with an exciton diffusion length of $9 \pm 3 \mathrm{~nm}$ and an intrastack energy-transfer rate constant of $10^{11}-10^{12} \mathrm{~s}^{-1}$. From fluorescence anisotropy decay measurements, we conclude that neighboring stacks aggregate in a herringbone structure, forming ordered domains that are randomly oriented in the substrate plane. These measurements indicate an interstack energy-transfer rate constant of $(7 \pm 2) \times 10^{10} \mathrm{~s}^{-1}$.
\end{abstract}

\section{Introduction}

This paper reports the results for singlet excitation transport of the first excited singlet state $\left(\mathrm{S}_{1}\right)$ in 5-25-nm-thick, selforganized films of zinc tetra-( $p$-octylphenyl)-porphyrin (ZnTOPP) (Figure 1) spin-coated onto quartz slides by doping with quenchers. The transport processes were investigated using steady-state as well as time-resolved fluorescence spectroscopy.

The preceding paper treats the photophysical properties of excitons of the second excited state $\left(\mathrm{S}_{2}\right)$. Self-organizing molecular materials, especially those forming a discotic liquidcrystalline phase, represent an interesting class of materials for photophysical studies. Excitation and charge carrier transport in crystalline materials have been widely studied, ${ }^{1-4}$ but considerably less work has been done on liquid-crystalline materials, thin films, and nanolayers. ${ }^{5-9}$ Several tetraphenylporphyrin derivatives self-organize into ordered aggregates. The liquid-crystalline phase of ZnTOPP has been reported to be lamellar discotic. Very recently, singlet and triplet exciton diffusion has been studied in 50-nm-thick, discotic layers of its free-base analog. ${ }^{10}$

At room temperature, organic materials usually exhibit localized (Frenkel) excitons. Excitation transport then occurs by incoherent diffusion or hopping. ${ }^{11-14}$ For liquid-crystalline perylene bis-(phenethylimide) films, it has been suggested that coherent energy transfer plays a significant role in the exciton motion. ${ }^{15}$ Also, several J-aggregates have been reported to have delocalized excitons at room temperature showing superradiance. ${ }^{16-18}$

The efficiency of exciton and charge transport is expected to depend on the ordering of these materials, e.g., the size of the

* Author to whom correspondence should be addressed. Present address: Laboratory for Inorganic Chemistry, Delft University of Technology, Delft, The Netherlands. Phone: +31-15-2783891. Fax: +31-15-2788047. E-mail: H.Donker@tnw.tudelft.nl.

$\dagger$ Present address: ASML Netherlands B.V., Veldhoven, The Netherlands.

$\doteqdot$ On leave from the Department of Systems Analysis, Belarusian State University, 4 F. Scoryna Ave., Minsk 220050, Belarus.

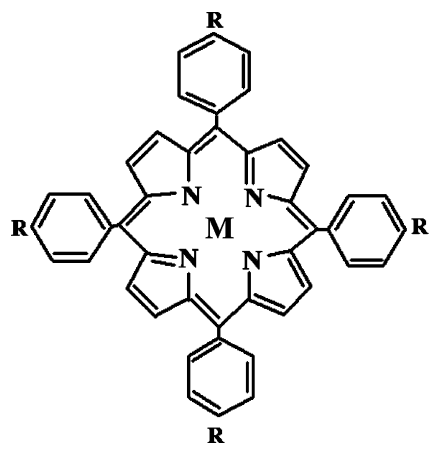

Figure 1. Molecular structure of zinc tetra-(p-octylphenyl)-porphyrin. $\mathrm{M}=\mathrm{Zn}, \mathrm{Cu}$, or $2 \mathrm{H}$ for $\mathrm{ZnTOPP}, \mathrm{CuTOPP}$, and $\mathrm{H}_{2} \mathrm{TOPP}$ free base. $\mathrm{R}=-\mathrm{C}_{8} \mathrm{H}_{17}$.

aggregates and the distances between and the relative orientation of the molecules with respect to the substrate and within the aggregate. The relevance of long-range order has been demonstrated for liquid-crystalline porphyrin sandwiched between two indium-tin oxide electrodes exhibiting a photovoltaic effect that strongly increases with the degree of order. ${ }^{19-21}$ However, ordering of a self-organizing free-base porphyrin on a $\mathrm{TiO}_{2}$ substrate has been found to decrease the singlet and triplet exciton diffusion as well as the incident photon to charge separation efficiency (IPCSE). ${ }^{10}$

The major causes for limiting the lifetime of excitons in organic films are the intrinsic lifetime of the excited state and trapping by impurities. The probability for trapping depends on the dimensionality of the diffusion process. For a given number of exciton jumps, the number of distinct sites visited by the exciton is smallest for one-dimensional diffusion, and therefore, for equal impurity concentrations, the probability of the exciton encountering an impurity in a layer consisting of linear stacks is smaller than that for two- or three-dimensional diffusion. ${ }^{22}$ Self-organized discotic dye molecules are interesting candidates for such layers, since many liquid-crystalline phases of discotic molecules show columnar ordering ${ }^{23}$ 
Depending on the properties of the film, small molecules from the surrounding atmosphere, e.g., oxygen and water vapor, ${ }^{24}$ can easily diffuse in and out, affecting the film ordering and trap densities. Interestingly, the absorption spectrum of ZnTOPP films is insensitive to atmospheric moisture, in contrast to films of zinc tetraphenylporphyrin (ZnTPP), which lack octyl substituents.

Monte Carlo simulations of excitation transport have been applied to the same ZnTOPP nanolayers using preliminary results of the present work. ${ }^{25}$

\section{Experimental Section}

Thin ZnTOPP films of up to several tenths of nanometers in thickness doped with various concentrations $(0.02-5 \mathrm{~mol} \%)$ of free-base tetra-(octylphenyl)-porphyrin $\left(\mathrm{H}_{2} \mathrm{TOPP}\right)$ or copper tetra-(octylphenyl)-porphyrin (CuTOPP) (Figure 1) as well as undoped films, both on quartz plates (Suprasil, $\varnothing 15 \mathrm{~mm}, 1-\mathrm{mm}$ thickness) were prepared by spin-coating at $2500 \mathrm{rpm}$ from $1-5$ $\mathrm{mM}$ toluene, pyridine, or chloroform solutions. Before spincoating, the quartz plates were subsequently rinsed with aqua regia, water, methanol, and toluene and blown dry with nitrogen. Through the use of the optical density of the films at $550 \mathrm{~nm}$, their estimated thicknesses were 5-25 $\pm 5 \mathrm{~nm}$, respectively. For the thickest film $(25 \mathrm{~nm})$, the amount of deposited porphyrin was also determined by dissolving the film in a known volume of toluene. Through the use of the extinction coefficient, ${ }^{26} \epsilon_{550}$ $=22000 \mathrm{~L} \mathrm{M} \mathrm{cm}^{-1}$, the calculated thickness was in good agreement with the above-mentioned values and corresponds to $\sim 100$ porphyrin layers for the investigated film. Since the appropriate amounts of ZnTOPP, $\mathrm{H}_{2}$ TOPP, and CuTOPP were first dissolved, it may be safely assumed that in the solutions the porphyrin molecules are statistically distributed. Since only the center of the porphyrins is different, we assume that the $\mathrm{H}_{2}$ TOPP and CuTOPP molecules are also distributed statistically among the ZnTOPP molecules in the spin-coated films. All solvents were p.a. quality, unless stated otherwise. Films of the analogous compounds without alkyl chains, i.e., tetraphenyl porphyrins (TPP), were used as references.

ZnTOPP/ZnTPP and CuTOPP were prepared by metallization of $\mathrm{H}_{2} \mathrm{TOPP} / \mathrm{H}_{2} \mathrm{TPP}$ by refluxing in dimethylformamide (DMF) with $\mathrm{ZnCl}_{2}$ (Merck, p.a.) and $\mathrm{CuCl}_{2} \cdot 2 \mathrm{H}_{2} \mathrm{O}$ (Merck, p.a.), respectively. ${ }^{27} \mathrm{H}_{2}$ TOPP and $\mathrm{H}_{2}$ TPP were synthesized by condensation of 4-(n-octyl)benzaldehyde and benzaldehyde (Kodak, 99\%), respectively, with pyrrole (Janssen Chimica, 99\%) in refluxing propionic acid (Merck, z.s.). ${ }^{28,29}$ The porphyrins were purified by chromatography on silica (Merck, silica gel 60) with toluene or chloroform as the eluent. For duplicate samples, ZnTOPP purchased from Porphyrin Products was used. All porphyrins are estimated to be $>99 \%$ pure as shown by thinlayer chromatography, absorption, and fluorescence spectroscopy.

Throughout this paper, films spin-coated from toluene are named films 1, and those spin-coated from chloroform films 2 .

Fluorescence spectra were recorded at room temperature using a Spex Fluorolog 3-22 fluorometer equipped with a 450-W xenon lamp as an excitation source. Spectra were measured in the front-face mode at an angle close to $0^{\circ}$ or in the right-angle mode at $\sim 45^{\circ}$ between the normal to the substrate and the incident light beam. Excitation spectra were corrected for lamp output, and emission spectra were corrected for photomultiplier sensitivity. Both types of spectra were also corrected for the monochromator transmission. Low-temperature $(1.4 \mathrm{~K})$ fluorescence measurements were carried out using a homemade liquid helium bath cryostat and a continuous wave (CW) $\mathrm{Ar}^{+}$ ion laser (Coherent CR-5) as an excitation source. Emission light was selected with an OG 540 cutoff filter and a JobinYvon HR1000 monochromator and detected with an S20 (EMI) photomultiplier.

Time-resolved fluorescence measurements were carried out using a mode-locked CW Nd:YLF laser (Coherent model Antares 76-YLF), equipped with an LBO frequency doubler with a temperature controller (Coherent model 7900 SHGTC) and a $\beta$-barium borate (BBO) frequency tripler (Coherent model 7950 THG), yielding up to 1-W CW mode-locked output power at $355 \mathrm{~nm}$. This UV light synchronously pumped a CW dye laser, using Stilbene 420 (Exciton Inc.) as a dye for excitation at 440 and $450 \mathrm{~nm}$ and Coumarin 460 for excitation at $465 \mathrm{~nm}$. The dye laser was a Coherent Radiation model CR 590, with a standard three-mirror-cavity design, but the output mirror was placed at the correct distance for perfect matching of the cavity lengths of the pump and dye laser. The high reflector pump and fold mirror had radii $R=75 \mathrm{~mm}$, whereas the high reflector end mirror radius was $R=50 \mathrm{~mm}$. The flat output mirror had a transmission of $\sim 8 \%$ over the $415-465 \mathrm{~nm}$ tuning range. Provisions were made to reduce the effective temperature dependence of the cavity length of the dye laser to a negligible value. To decrease the excitation pulse rate to $594 \mathrm{kHz}$, electrooptic modulators were used in a dual pass configuration. ${ }^{30}$ The excitation pulse width was $\sim 4$ ps full width at halfmaximum (fwhm) at a maximum pulse energy of about 100 pJ. Spin-coated films were fixed on a thermostated, springloaded holder. Extreme care was taken to avoid artifacts from depolarization effects by the following procedure: At the front of the sample housing, a Glan laser polarizer was mounted, further optimizing the already vertical polarization of the input light beam. The reflections and the scatter of the excitation beam incident on the substrate were caught on highly absorbing black paper to prevent multiple excitation of the sample. The sample holder was placed in a housing also containing part of the detection optics. The fluorescence was collected at an angle of $90^{\circ}$ with respect to the direction of the exciting light beam. Between the sample and the photomultiplier detector were placed a set of single fast lenses (uncoated fused silica, F/3.0) with a rotatable sheet-type polarizer between them, followed by a monochromator and a second set of single fast lenses (uncoated fused silica, F/3.0), focusing the output light of the monochromator on the photomultiplier cathode. The polarizer sheet was in a direct current (dc)-motor-driven ball-bearing holder with mechanical stops, allowing computer-controlled rotation $(0.2 \mathrm{~s})$. The sheet polarizer was Polaroid type HNP'B. The detection monochromator was a CVI model Digikröm 112 double monochromator (F/3.9) with the two gratings placed in a subtractive dispersion configuration. The relative detection sensitivities of the monochromator at different wavelengths for horizontally and perpendicularly polarized light ( $G$-factors) were determined by using unpolarized and depolarized light from a glow bulb or by detecting the decay of a fast rotating fluorescence probe and applying a so-called tail-matching procedure. Polarizer positions were carefully aligned, and the setup performance was finally checked by measuring reference samples.

Detection electronics were standard time-correlated singlephoton-counting modules. The start signal for the time-toamplitude converter (TAC, Tennelec model TC 864) was generated by using one channel of a quad constant fraction discriminator (CFD, Tennelec modified model TC 454), driven via a wide-band amplifier (Becker \& Hickl model ACA $35 \mathrm{~dB}$, $1.8 \mathrm{GHz}$ ) by the pulses from a fast PIN photodiode (Hewlett- 
Packard model 5082-4204 at 45-V reverse bias), excited with a small fraction of the intensity of the exciting light pulses. Single fluorescence photon responses from a microchannel plate photomultiplier (Hamamatsu model R1645U-01 at $2800 \mathrm{~V}$ or R3809U-50 at $3100 \mathrm{~V}$ ) were amplified by a wide-band amplifier (Hewlett-Packard model 8847 F or Becker \& Hickl model ACA-2 $21 \mathrm{~dB}, 1.8 \mathrm{GHz}$ ), analyzed in another channel of the CFD and then used as the stop signal for the TAC. The output pulses of the TAC were analyzed by an analog-to-digital converter (ADC, Nuclear Data model 8715, 800 ns fixed dead time), used in coincidence and sampled voltage analysis mode, triggered by the valid conversion output pulses of the TAC. The output of the ADC was collected in 1024 channels of a multichannel analyzer (MCA board from Nuclear Data model AccuspecB in a PC).

By reduction of the intensity of the excitation pulses, a maximum photon frequency of $30 \mathrm{kHz}(\sim 5 \%$ of $594 \mathrm{kHz})$ was chosen $^{31}$ to prevent pile-up distortion. Also other instrumental sources for distortion of data were minimized ${ }^{32}$ to below the noise level of normal photon statistics. By maximizing the sensitivity of the fluorescence setup, we made certain that excitation and detection involve only single-photon processes. Without these precautions, it should not come as a surprise that the fluorescence decays do not reproduce correctly due to contamination by artifacts. Also, we took extreme care to prevent artifacts from background luminescence. All substrates were carefully cleaned and checked for background luminescence prior to the measurements. For samples with a low fluorescence yield, the background luminescence of an uncoated substrate was recorded and subtracted from the sample data in analysis. Fluorescence decay curves were measured with the emission polarizer at the magic angle. Fluorescence anisotropy decay curves were obtained with an angle of $25^{\circ}$ between the sample and the direction of the excitation beam. To obtain a dynamic instrumental response for deconvolution purposes, the scatter of a rough-hewn, uncoated quartz substrate of 1-mm thickness was measured at the excitation wavelength. Data analysis was performed using a home-built program (Fluorescence Data Processor, version 1, developed at the Systems Analysis Department of the Belarusian State University in Minsk, Belarus). ${ }^{33}$

\section{Results and Discussion}

Steady-State Fluorescence. Figure 2 presents the roomtemperature fluorescence emission and excitation spectra of undoped ZnTOPP and $\mathrm{H}_{2}$ TOPP films. The excitation spectra are very similar to the absorption spectra and do not contain additional bands. The emission spectra of undoped ZnTOPP films contain four bands viz. at 560, 590, 641, and $711 \mathrm{~nm}$. The emission and excitation spectra in the Q-band region are similar to those of a ZnTOPP solution, indicating that in comparison with the strong interactions between the $S_{2}$ states, as described in the preceding paper, the excitonic interaction for the $S_{1}$ states is much weaker, resulting in localized $S_{1}$ excitons. As for most organic materials, their motion can properly be described as incoherent diffusion or hopping. ${ }^{11-14}$ Whereas the 590 and $641 \mathrm{~nm}$ bands can be assigned to the $(0,0)$ and $(0,1)$ emission of ZnTOPP, the origin of the weak $711 \mathrm{~nm}$ band is not completely clear, since it may be due to either the $(0,2)$ emission of ZnTOPP or the $(0,1)$ emission of a trace amount of $\mathrm{H}_{2}$ TOPP, resulting from incomplete metallization during preparation from the $\mathrm{H}_{2}$ TOPP precursor. The very weak

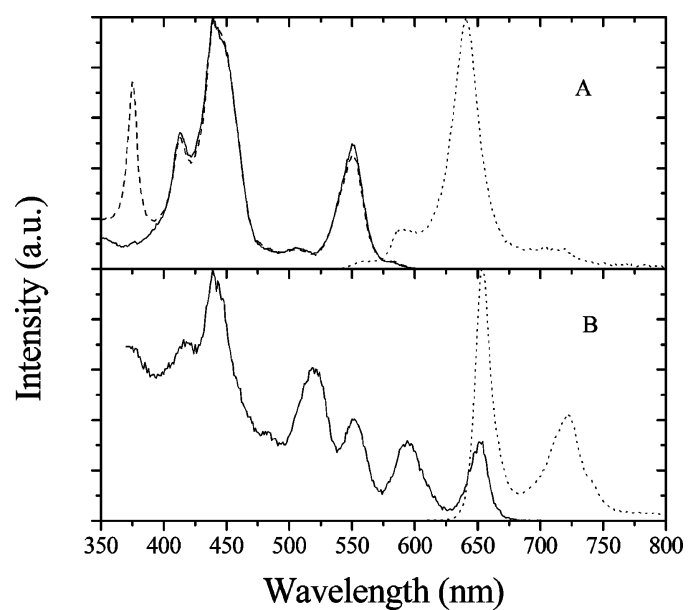

Figure 2. (a) Room-temperature emission and excitation spectra of an undoped ZnTOPP film: solid line, excitation spectrum at normal incidence, $\lambda_{\mathrm{em}}=640 \mathrm{~nm}$; dashed line, excitation spectrum at $45^{\circ}$ angle of incidence, $\lambda_{\mathrm{em}}=640 \mathrm{~nm}$; dotted line, emission spectrum, $\lambda_{\mathrm{exc}}=$ $450 \mathrm{~nm}$. (b) Room-temperature emission and excitation spectra of an undoped $\mathrm{H}_{2}$ TOPP film: solid line, excitation spectrum at $45^{\circ}$ angle of incidence, $\lambda_{\mathrm{em}}=720 \mathrm{~nm}$; dotted line, emission spectrum, $\lambda_{\mathrm{exc}}=450$ $\mathrm{nm}$.

$560 \mathrm{~nm}$ band is probably a $(1,0)$ hot band emission of ZnTOPP, in agreement with its absence in emission spectra at $T<200$ $\mathrm{K}$.

In the room-temperature emission spectrum of an undoped $\mathrm{H}_{2}$ TOPP film, two emission bands are observed at 655 and 722 $\mathrm{nm}$, which are ascribed to the $(0,0)$ and $(0,1)$ transitions of $\mathrm{H}_{2-}$ TOPP, respectively. At $\sim 610 \mathrm{~nm}$, a very weak shoulder (not visible in Figure 2) is observed on the $655 \mathrm{~nm}$ band. This shoulder is probably due to the $(1,0)$ hot band emission of $\mathrm{H}_{2-}$ TOPP.

Figures $3 \mathrm{~A}$ and $3 \mathrm{~B}$ show the $1.4 \mathrm{~K}$ fluorescence spectra of ZnTOPP before (A) and after (B) exposure to chloroform vapor and the difference spectrum $(\mathrm{C})$ after normalizing the intensities of parts $\mathrm{A}$ and $\mathrm{B}$ at $585 \mathrm{~nm}$. Figure $3 \mathrm{~A}$ contains two dominant $\mathrm{Q}(0,0)$ and $\mathrm{Q}(0,1)$ bands at 584 and $642 \mathrm{~nm}$, respectively, with $I_{\mathrm{Q}(0,0)} / I_{\mathrm{Q}(0,1)}<1$. There are only minor differences between the fluorescence spectra of Figure $3 \mathrm{~B}$ and those of ZnTOPP or ZnTPP spin-coated from chloroform solution (not shown). For the difference spectrum of Figure $3 \mathrm{C}$ of Figures $3 \mathrm{~B}$ and $3 \mathrm{~A}$, $I_{\mathrm{Q}(0,0)} / I_{\mathrm{Q}(0,1)}>1$. The fluorescence spectrum of ZnTOPP spincoated from chloroform was found to be a superposition of the spectra of Figure $3 \mathrm{~A}$ and $3 \mathrm{C}$ but with a variable intensity ratio, depending on the film history.

The room-temperature fluorescence spectra of films 1 do not show a noticeable change upon exposure to chloroform vapor, indicating that chloroform results in a relatively weak ligation of $\mathrm{ZnTOPP}$, in contrast with the spectral effects of exposure to a strong ligand such as pyridine. ${ }^{34-36}$ At low temperatures, the chloroform treatment results in a significantly different fluorescence spectrum (Figures 3A-3C), however. Upon comparison of the fluorescence spectra of Figures $3 \mathrm{~A}$ and $3 \mathrm{C}$ with published data, ${ }^{34}$ the additional emission bands of Figure $3 \mathrm{~B}$ can be identified as due to ligated zinc porphyrin monomers. In contrast with their low-temperature fluorescence spectra, the absorption spectra of films $\mathbf{1}$ and $\mathbf{2}$ are found to be identical at room temperature as well as at 80 and $1.4 \mathrm{~K}$, clearly indicating that the fluorescence of films exposed to chloroform originates from traps, whereas the absorption spectra reflect the properties of the bulk material. From the energy difference between the zero phonon lines of the ligated and nonligated ZnTOPP, a trap depth $\Delta \approx 140 \mathrm{~cm}^{-1}$ is calculated. At room temperature, $\Delta<$ 


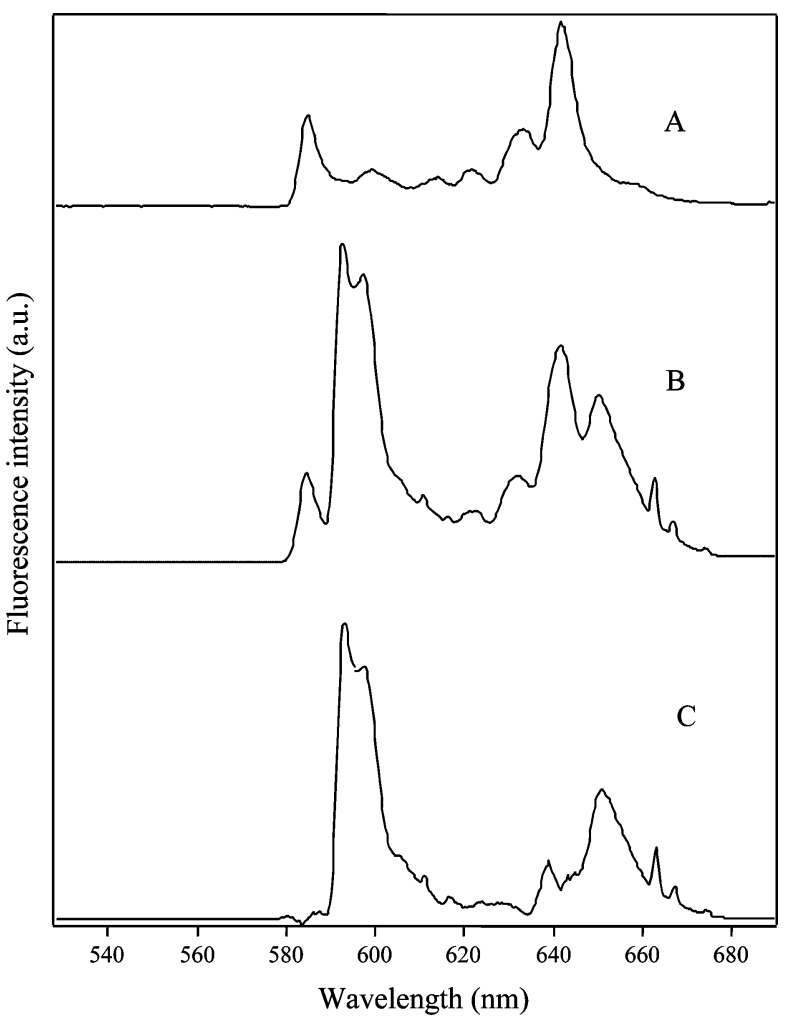

Figure 3. Fluorescence spectra, excited by the $\mathrm{Ar}^{+} 357.9 \mathrm{~nm}$ line, at $1.4 \mathrm{~K}$ of (A) film 1, (B) film 1 exposed to chloroform vapor, and (C) the difference of normalized spectra $\mathrm{A}$ and $\mathrm{B}$.

$T$, explaining why these traps do not show up in the roomtemperature emission spectra.

We now focus on ZnTOPP films doped and undoped with $\mathrm{H}_{2} \mathrm{TOPP}$ spin-coated from toluene only. The excitation spectra detecting the $\mathrm{H}_{2}$ TOPP fluorescence emission in the mixed $\mathrm{ZnTOPP} / \mathrm{H}_{2}$ TOPP films are similar to the ZnTOPP excitation spectrum in an undoped film, implying efficient energy transfer from $\mathrm{ZnTOPP}$ to $\mathrm{H}_{2}$ TOPP. Since these transitions are allowed, the dominant interaction is of the dipole-dipole type. From the spectra of undoped ZnTOPP and $\mathrm{H}_{2}$ TOPP films, the spectral overlap between the ZnTOPP fluorescence spectrum and the $\mathrm{H}_{2}$ TOPP and ZnTOPP absorption spectra has been determined to be 2.65 and $0.14 \mathrm{eV}^{-1}$, respectively. From this large difference in spectral overlap and noting that all other factors determining the dipole-dipole energy-transfer rate are approximately equal, we conclude that for nearest neighbors in a stack the ZnTOPP to $\mathrm{H}_{2}$ TOPP energy-transfer probability is much larger than that for ZnTOPP to ZnTOPP energy transfer. Since also the lowest singlet level of $\mathrm{H}_{2} \mathrm{TOPP}$ is at a lower energy than that of ZnTOPP, $\mathrm{H}_{2}$ TOPP is an efficient exciton trap. However, if we assume that the dominant interaction is dipole-dipole coupling, then its $1 / r^{6}$ dependence means that for an excited ZnTOPP molecule with the closest $\mathrm{H}_{2}$ TOPP molecule on the next nearest neighbor position in the stack the probability that energy transfer occurs first from the excited ZnTOPP to the neighboring ground-state ZnTOPP molecule and next to $\mathrm{H}_{2}$ TOPP is much larger than that of direct transfer to this more distant $\mathrm{H}_{2}$ TOPP. Thus, energy transfer mainly occurs by exciton diffusion, as we have argued before, followed by trapping. The rate-limiting step in this process is exciton diffusion; i.e., energy transfer occurs by diffusion-limited energy migration. Energy migration by diffusion has previously been reported in films of octaethylporphyrin. ${ }^{37-39}$

Models describing diffusion-limited energy migration usually assume infinitely deep traps. Fluorescence decay measurements

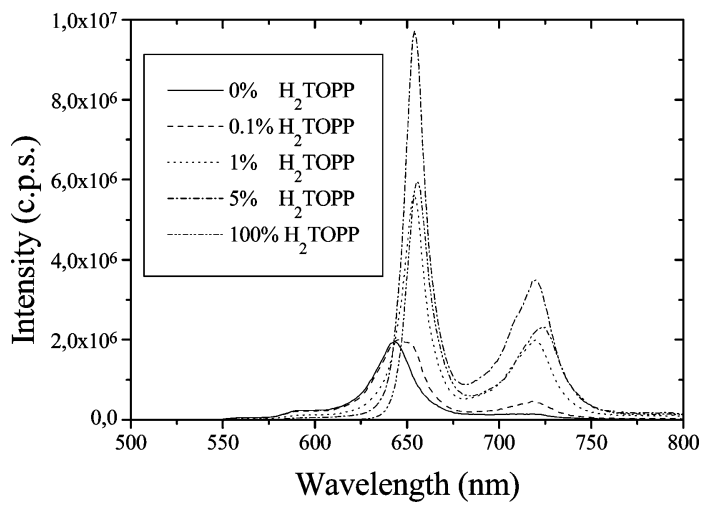

Figure 4. Emission spectra of ZnTOPP films doped with various concentrations of $\mathrm{H}_{2}$ TOPP as well as of undoped films using excitation in the B-band $\left(\lambda_{\text {exc }}=450 \mathrm{~nm}\right)$.

(see below), however, show that after trapping $\mathrm{H}_{2}$ TOPP to ZnTOPP back-transfer may occur, complicating the analysis of the fluorescence decay curves.

To determine how important back-transfer is in these doped films, we investigated $\mathrm{Cu}$ (II)TOPP-doped ZnTOPP films, since nonfluorescent $\mathrm{Cu}(\mathrm{II})$ porphyrins are well-known efficient quenchers of the fluorescence of porphyrins. For these films, no CuTOPP to ZnTOPP back-transfer is observed. CuTOPP has the drawback that its quenching mechanism is not well understood, ${ }^{40-42}$ but a similar concentration dependence of the fluorescence quenching as with $\mathrm{H}_{2} \mathrm{TOPP}$ is observed, indicating that also for CuTOPP-doped films ZnTOPP to ZnTOPP exciton diffusion is the rate-limiting step.

Figure 4 shows the emission spectra of ZnTOPP films doped with various $\mathrm{H}_{2}$ TOPP concentrations upon excitation in the ZnTOPP B-band. With increased $\mathrm{H}_{2}$ TOPP concentrations, the intensity of the $\mathrm{H}_{2}$ TOPP emission increases at the expense of the ZnTOPP emission intensity, as expected since the average distance between $\mathrm{ZnTOPP}$ and $\mathrm{H}_{2}$ TOPP molecules decreases at increasing $\mathrm{H}_{2}$ TOPP concentrations, facilitating energy transfer. In an undoped ZnTOPP film, an exciton can either decay after reaching a certain ZnTOPP molecule or be trapped followed by decay at a nonintentional chemical impurity or lattice defect. ZnTOPP may form part of the traps with a different conformation or environment than that of the unperturbed ZnTOPP molecules. In a ZnTOPP film intentionally doped with $\mathrm{H}_{2}$ TOPP or CuTOPP, the excitons can of course also diffuse to these doped lattice sites, where they are trapped and then decay. For the present analysis, we assume that the emission of an undoped ZnTOPP film mainly originates from unperturbed or only slightly perturbed ZnTOPP molecules. It is most likely that the latter form shallow traps with a distribution of trap depths, such that at room temperature exciton diffusion is not stopped. Then, in a doped film, ZnTOPP to $\mathrm{H}_{2}$ TOPP energy transfer readily occurs.

In the preceding paper, we have concluded from the absorption spectra that ZnTOPP films are built from linear slippeddeck-of-cards stacks, as has also been found for a number of octyl-substituted porphyrins. ${ }^{43-46}$ The distance between two molecules in a stack is estimated to be $\sim 0.5 \mathrm{~nm}$, much shorter than the interstack distance, estimated to be $1-5 \mathrm{~nm}$, determined by the porphyrin diameter. The probability for energy transfer within a stack is therefore expected to be much larger than that between stacks; i.e., the exciton diffusion is assumed to be onedimensional. Fluorescence decay and anisotropy decay measurements (see below) show that the exciton diffusion is not strictly one-dimensional but also that the probability for interstack energy transfer is at least an order of magnitude smaller than 


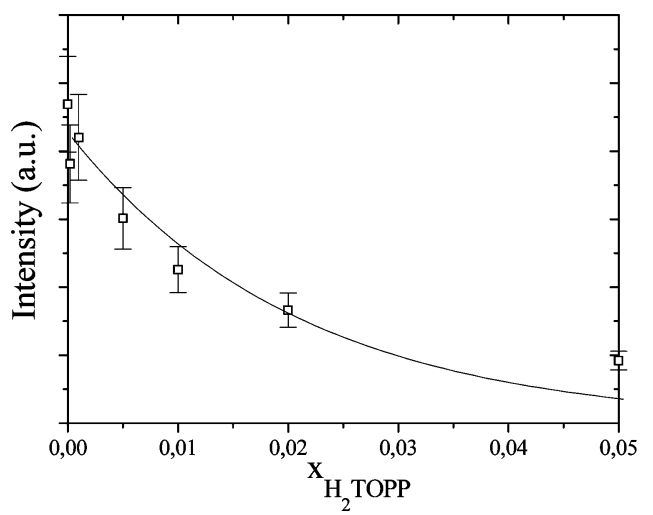

Figure 5. ZnTOPP emission intensity at $590 \mathrm{~nm}$ vs the $\mathrm{H}_{2}$ TOPP mole fraction. Solid line is the best fit to eq 4 .

that for intrastack transfer and is therefore neglected in the following discussion.

The number of jumps $(n)$ that an exciton makes during its lifetime equals the product of the jump frequency $(v)$ and the exciton lifetime $\left(\tau_{\text {exc }}\right)$. The average number of distinct sites visited during a one-dimensional random walk can be approximated for large $n$ by ${ }^{22}$

$$
S \approx \sqrt{\left(\frac{8 n}{\pi}\right)}=\sqrt{\left(\frac{8 v \tau_{\mathrm{exc}}}{\pi}\right)}
$$

Using the Einstein-Smoluchowski relation $D=v l^{2} / 2$ where $l$ is the intermolecular distance leads to

$$
S \approx \sqrt{\left(\frac{16 D_{\mathrm{exc}} \tau_{\mathrm{exc}}}{\pi l^{2}}\right)}=\frac{4}{\sqrt{\pi}} \frac{L_{\mathrm{exc}}}{l}
$$

Assuming that the exciton diffusion is the rate-limiting step for trapping and that an exciton is trapped on its first encounter with a trap, the number of excitons that will survive is approximately proportional to the probability that the average number of distinct sites visited during its lifetime does not contain a trap, i.e., $(1-x)^{S}$, where $x$ is the mole fraction of traps. This implies that the dependence of the intensity of the intrinsic ZnTOPP emission $\left(I_{\mathrm{Zn}}\right)$ on the trap mole fraction can be approximated by

$$
I_{\mathrm{Zn}}=I_{\mathrm{Zn}, 0}(1-x)^{S}=I_{\mathrm{Zn}, 0}(1-x)^{(4 / \sqrt{\pi})\left(L_{\mathrm{exc}} l\right)}
$$

ZnTOPP films also contain nonintentionally incorporated traps. Assuming that the trapping efficiency of all traps is the same, the mole fraction of traps $x$ can be split into a fraction of nonintentional traps $\left(x_{0}\right)$ and a fraction of intentional traps $\left(x_{\mathrm{H}_{2}}\right)$. Then

$$
I_{\mathrm{Zn}}\left(x_{\mathrm{H}_{2}}\right)=I_{\mathrm{Zn}, 0}\left[1-\left(x_{0}+x_{\mathrm{H}_{2}}\right)\right]^{S}
$$

where $I_{\mathrm{Zn}, 0}$ is the emission intensity of a pure ZnTOPP film. We have used values between 0 and 0.01 for $x_{0}$ to obtain the exciton diffusion length from Figure 4. In this range, the result of the fit turns out to be rather insensitive to the value of $x_{0}$.

Figure 5 shows a plot of the emission intensity at $590 \mathrm{~nm}$ versus the $\mathrm{H}_{2}$ TOPP concentration. Since no $\mathrm{H}_{2} \mathrm{TOPP}$ emission is observed at this wavelength, this intensity can be taken as a measure for the total ZnTOPP emission intensity. The solid line in Figure 5 is the best fit of the $590 \mathrm{~nm}$ data to eq 4 with $x_{0}=$ 0.005 , yielding $L_{\mathrm{exc}} / l=18 \pm 6$. Assuming that the distance $l$

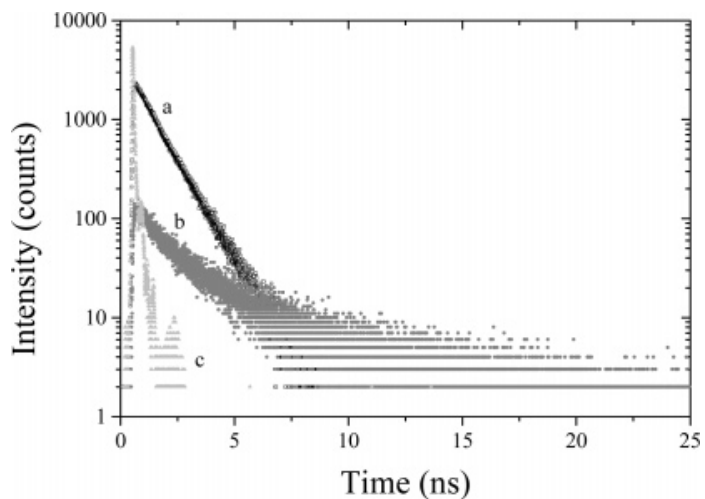

Figure 6. Fluorescence decay curves for a ZnTOPP film: (a) $\lambda_{\mathrm{em}}=$ $580 \mathrm{~nm}$; (b) $\lambda_{\mathrm{em}}=725 \mathrm{~nm}$; (c) instrumental response at $465 \mathrm{~nm}$. $\left(\lambda_{\text {exc }}=465 \mathrm{~nm}, 3.125 \mathrm{ps} / \mathrm{ch}\right)$.

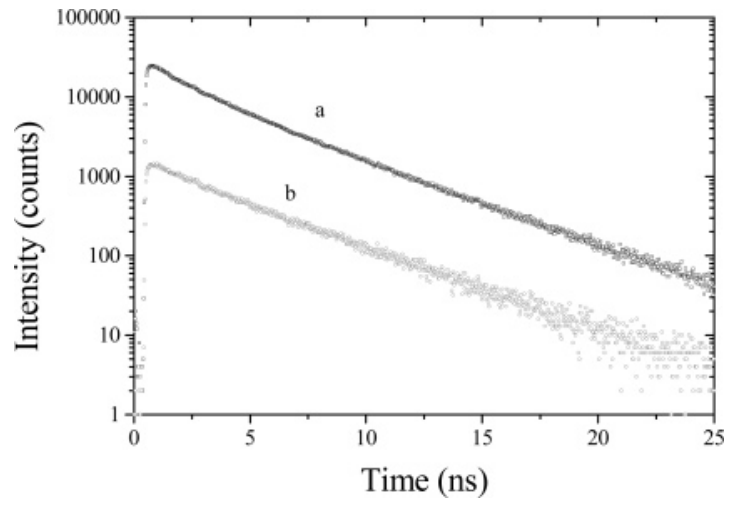

Figure 7. Fluorescence decay curves for a $\mathrm{H}_{2} \mathrm{TOPP}$ film: (a) $\lambda_{\mathrm{em}}=$ $640 \mathrm{~nm}$; (b) $\lambda_{\mathrm{em}}=725 \mathrm{~nm}$. ( $\left.\lambda_{\mathrm{exc}}=450 \mathrm{~nm}, 25 \mathrm{ps} / \mathrm{ch}\right)$.

between two molecules in the stack is $\sim 0.5 \mathrm{~nm}$, this results in an exciton diffusion length of $9 \pm 3 \mathrm{~nm}$.

In a previous paper, ${ }^{47}$ we compared this exciton diffusion length with that obtained from photocurrent response measurements, assuming a random orientation of one-dimensional aggregates. The polarized absorption measurements discussed before indicate, however, that the aggregates in the film are oriented perpendicular to the substrate, which makes a comparison between both methods unjustified. The correspondence between values obtained for the exciton diffusion length from both methods must therefore be considered as fortuitous.

Fluorescence Decay Measurements. Figure 6 shows the fluorescence decay curves for an undoped ZnTOPP film at 580 and $725 \mathrm{~nm}$. The two decay curves have different shapes, indicating that in the undoped films the $718 \mathrm{~nm}$ emission band is indeed not only due to ZnTOPP. The long decay at $725 \mathrm{~nm}$ is similar to that of $\mathrm{H}_{2}$ TOPP-doped films (Figure 7), indicating that the $718 \mathrm{~nm}$ emission contains a $\mathrm{H}_{2}$ TOPP component. Leastsquares fitting shows that the observed decay curve detected at $580 \mathrm{~nm}$ is not a single exponential. Figure 8 shows the decay curves for a ZnTOPP film intentionally doped with $1 \% \mathrm{H}_{2} \mathrm{TOPP}$ detected at the same wavelengths as in Figure 6. There are several striking differences with the decay curves of Figure 6:

- For the $580 \mathrm{~nm}$ decay curve, the initial decay is much steeper than that for the undoped film.

- This decay also contains a long, approximately monoexponential tail.

- The decay curve detected at $725 \mathrm{~nm}$ shows a slower buildup and only contains a slow component similar to the tail observed at $580 \mathrm{~nm}$.

The initial phase of the nonexponential decay at $580 \mathrm{~nm}$ is much steeper upon doping with $\mathrm{H}_{2}$ TOPP or CuTOPP (see 


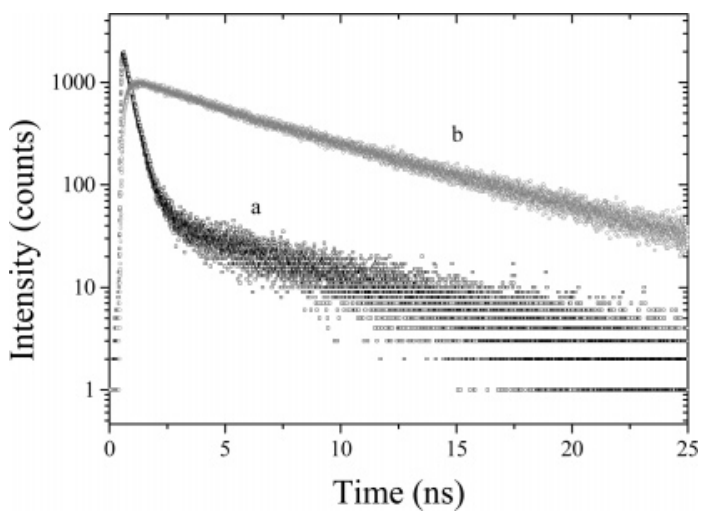

Figure 8. Fluorescence decay curves for a ZnTOPP film doped with $1 \% \mathrm{H}_{2}$ TOPP: (a) $\lambda_{\mathrm{em}}=580 \mathrm{~nm}$; (b) $\lambda_{\mathrm{em}}=725 \mathrm{~nm}$. $\left(\lambda_{\mathrm{exc}}=465 \mathrm{~nm}\right.$, $3.125 \mathrm{ps} / \mathrm{ch})$.

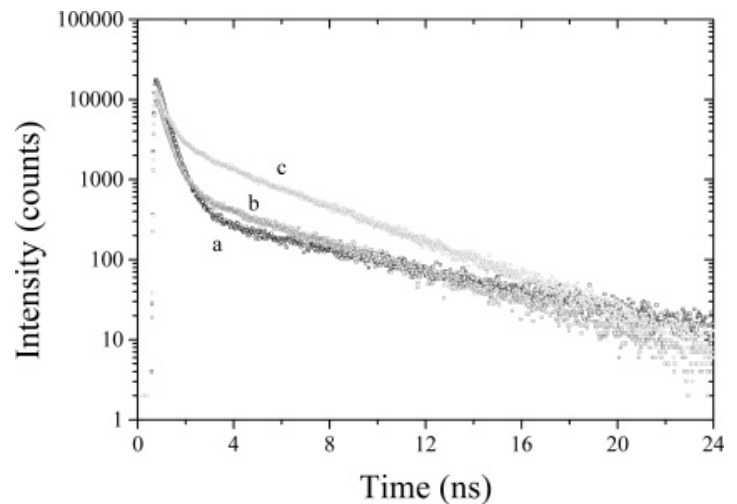

Figure 9. Fluorescence decay curve of a ZnTOPP film doped with $1 \% \mathrm{H}_{2}$ TOPP as a function of temperature: (a) $296 \mathrm{~K}$; (b) $333 \mathrm{~K}$; (c) $373 \mathrm{~K}$. $\left(\lambda_{\mathrm{em}}=580 \mathrm{~nm}, \lambda_{\mathrm{exc}}=450 \mathrm{~nm}, 24 \mathrm{ps} / \mathrm{ch}\right)$.

below). Combined with the spectral changes resulting from doping as discussed previously, we may conclude that energy transfer or migration occurs in the film. As shown below, the dependence of the decay curves on dopant concentration is inconsistent with direct energy transfer from ZnTOPP to $\mathrm{H}_{2-}$ TOPP or CuTOPP, implying that transfer must occur by energy migration through the ZnTOPP lattice. This process is most likely one- or two-dimensional, since for three-dimensional energy migration a monoexponential decay is predicted, ${ }^{1,2}$ in contradiction with the experimental results. Multiexponential decay detected at $580 \mathrm{~nm}$ of the undoped film results from the presence of nonintentional traps, most probably $\mathrm{H}_{2} \mathrm{TOPP}$.

Since the decay of the doped film is even longer than that of an undoped film, the long-decay component of $\mathrm{H}_{2}$ TOPP-doped films must be due to $\mathrm{H}_{2}$ TOPP. This is supported by the finding that at $580 \mathrm{~nm}$ the intensity of this tail increases with increasing $\mathrm{H}_{2}$ TOPP concentration. Also, the decay time of $\sim 7 \mathrm{~ns}$ associated with this tail equals that at $725 \mathrm{~nm}$. However, as can be seen in Figure 2, $\mathrm{H}_{2}$ TOPP does not show any emission at $580 \mathrm{~nm}$. Although wide slits $(\Delta \lambda=16 \mathrm{~nm})$ were used to measure the decay time, it is highly unlikely that we also monitored the $\mathrm{H}_{2-}$ TOPP emission at $580 \mathrm{~nm}$, since its fluorescence onset starts beyond $\sim 600 \mathrm{~nm}$. Furthermore, since also the decay measured at $560 \mathrm{~nm}$ is very similar to that at $580 \mathrm{~nm}$, we ascribe the long tail to $\mathrm{H}_{2}$ TOPP to ZnTOPP back-transfer.

Figure 9 shows the temperature dependence of the decay time. The intensity of the $725 \mathrm{~nm}$ emission decreases with increasing temperature, and its decay becomes faster. The $580 \mathrm{~nm}$ emission, however, increases with increasing temperature. As can be seen in this figure, this increase is mainly due to a higher intensity in the tail of the decay curve. The decay time associated with
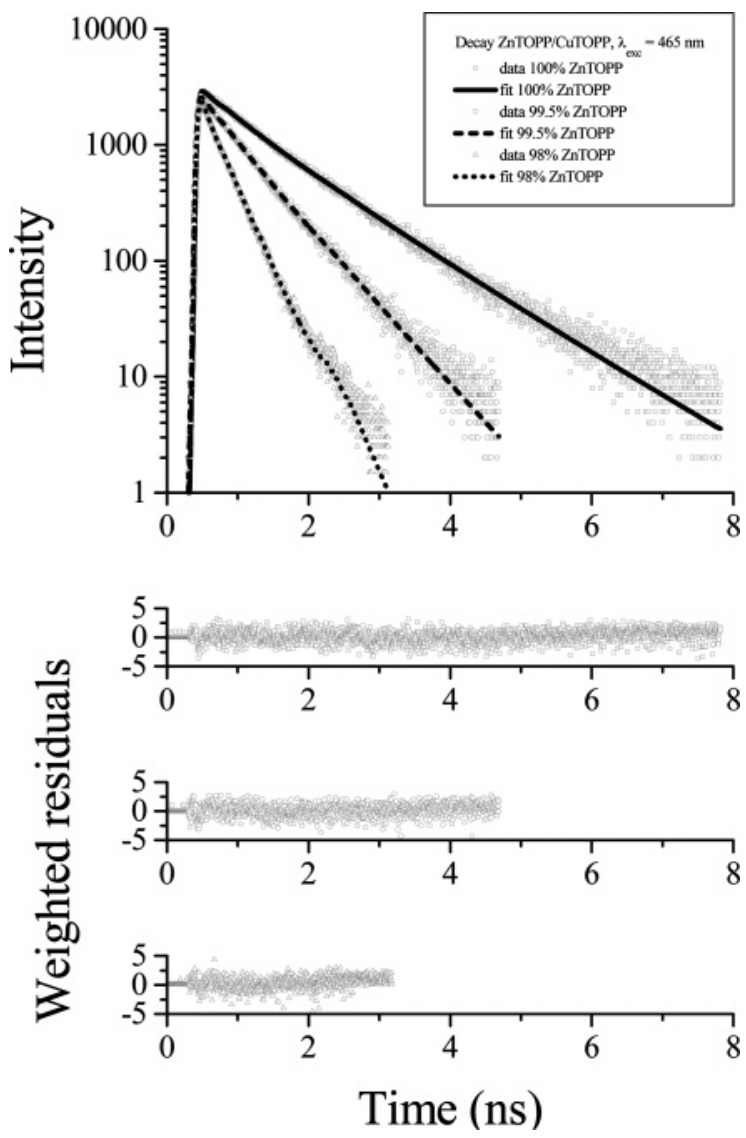

Figure 10. Analysis of fluorescence decay curves of ZnTOPP films doped with CuTOPP using eq 5. The drawn lines are the convolutions, using eq 5 with parameters obtained from least-squares fitting and the instrumental response function. Weighted residuals from top to bottom correspond to $\mathrm{a}-\mathrm{c}$.

this tail shows a temperature dependence very similar to that of the decay at $725 \mathrm{~nm}$. These temperature effects are in complete agreement with the results expected for thermally activated back-transfer.

The decay curves at $580 \mathrm{~nm}$ for CuTOPP-doped ZnTOPP shown in Figure 10 are similar to those of ZnTOPP films doped with $\mathrm{H}_{2}$ TOPP, but the intensity of the long tail is now at least 2 orders of magnitude smaller and not visible in this figure. That this tail has not completely disappeared is ascribed to a small nonintentional amount of $\mathrm{H}_{2}$ TOPP.

On the basis of the results of the preceding paper and the spectral data, we have discussed above that energy transfer will occur by exciton diffusion, and since distances between molecules within a stack are shorter than distances between stacks, this diffusion is expected to be one-dimensional. Two different models have frequently been used to describe onedimensional, diffusion-limited energy migration, i.e., the Movaghar, Sauer, and Würtz (MSW) ${ }^{48}$ and the Wieting, Fayer, and Dlott (WFD) ${ }^{49-51}$ models. The models have in common that the fluorescence decay can be described by

$$
I(t)=I_{0} \exp (-t / \tau) \exp \left(-k_{1} t^{1 / n}\right)
$$

The MSW model assumes that the energy migration is strictly one-dimensional and that the trapping is by infinitely deep traps. This model has especially been applied to fit the tail of decay curves. In that case, $n=3$ and $\tau$ is a constant, i.e., the decay time of a ZnTOPP molecule. This approximation is only valid for $I<0.01 I_{0}$, however, i.e., at relatively long times. ${ }^{52}$ For the doped films, the back-transfer from $\mathrm{H}_{2}$ TOPP molecules com- 
pletely dominates the decay curve in that region, and therefore, eq 5 cannot be applied. However, according to the MSW model, the initial part of the decay curve can also be described with eq 5 but with $n=2$, which equals the first passage time (FPT) approximation, ${ }^{53}$ where $k_{1}$ is given by

$$
k_{1}=4 x\left(\frac{W}{\pi}\right)^{1 / 2}
$$

with $x$ the mole fraction of traps and $W$ the donor-donor transfer rate.

The WFD model has also frequently been used to describe one-dimensional energy migration. By contrast with the MSW model, it also allows for the presence of a three-dimensional energy migration component, in fact constituting a quasi-onedimensional model. The intensity of the donor luminescence versus time is also given by eq 5 with $n=2$, but now $1 / \tau=$ $1 / \tau_{0}+k_{2}$, where $k_{2}$ is dependent on the trap concentration and reflects the three-dimensional energy migration. The $t^{1 / 2} \mathrm{de}-$ pendence is similar to that of the MSW model but is now due to one- as well as three-dimensional energy migration components. However, the WFD model is valid for the entire decay curve and not only the initial part as in the MSW model. For strictly one-dimensional migration, the time dependence for the initial decay is the same for the two models. A problem then is that the time and concentration dependence of the initial decay are similar to those expected for direct transfer in an isotropic three-dimensional system. ${ }^{54,55}$ However, as argued above, direct transfer is not likely to be the dominant mechanism for energy transfer in this system. Furthermore, the experimental results (see below) show that $\tau$ is dependent on the trap concentration, which is in contradiction with direct transfer of any dimensionality. 55,56

Another difference between the MSW and WFD models is that in the former infinitely long, one-dimensional arrays with only trapping impurities are assumed, whereas the WFD theory assumes the presence of trapping as well as scattering impurities, where the latter obstruct the one-dimensional exciton diffusion. This is only valid if the concentration of scattering impurities is larger than that of the trapping impurities. In other words, the MSW and WFD models are valid if the exciton diffusion length is shorter or longer than the stack length, respectively. Since for pure one-dimensional diffusion both models predict similar decay curves, it is not possible to determine which model yields the best fit to the experimental decay time curves.

Figure 10 shows the fluorescence decay at $580 \mathrm{~nm}$ for the first few nanoseconds for several ZnTOPP films doped with various concentrations of CuTOPP. The observed relative amount of back-transfer is so small that certainly in the first part of the decay curve it can be neglected. The drawn lines in Figure 10 are the best fits to eq 5 with $n=2$.

Both parameters $\tau$ and $k_{1}$ are found to vary with the CuTOPP concentration as shown in Figure 11. The parameters for $\mathrm{H}_{2}$ TOPP-doped ZnTOPP films are similar but less accurate due to back-transfer. This figure clearly shows that $\tau$ varies with the dopant concentration, and thus it can be concluded that the migration in the films is not strictly one-dimensional but quasione-dimensional.

According to the WFD model, both $1 / \tau$ and $k_{1}$ should vary linearly with the trap concentration, as is indeed found (cf. Figure 11). The value of $k_{1}$ is mainly determined by the initial part of the decay curve, however. Due to an increase of the three-dimensional component, this value becomes increasingly less accurate with increasing trap concentration. Through the use of eq 6 and the obtained $k_{1}$ values, $W$ can be estimated to

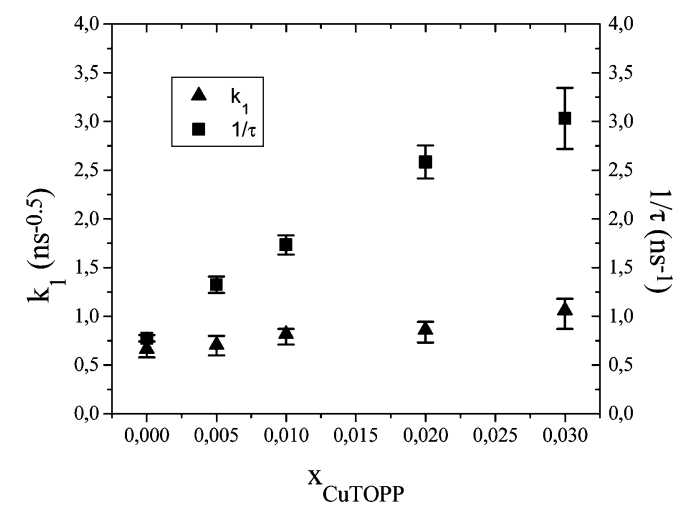

Figure 11. Fitting parameters $k_{1}$ and $1 / \tau$ (eq 5) vs the CuTOPP mole fraction.

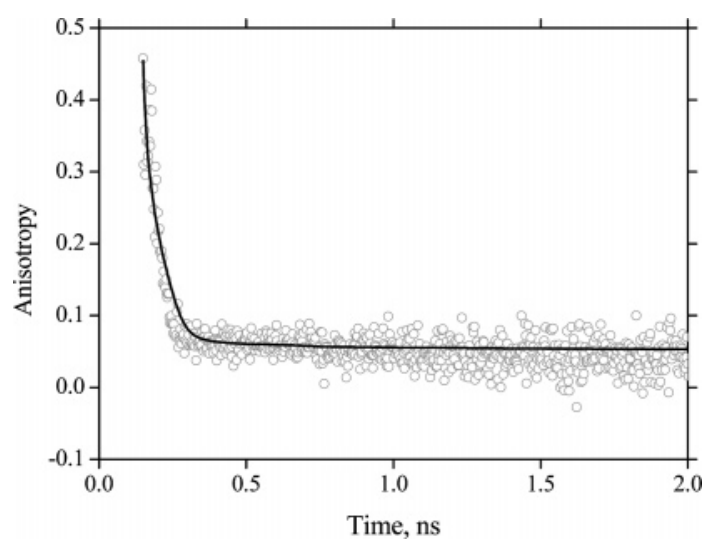

Figure 12. Fluorescence anisotropy decay of a ZnTOPP film spincoated from toluene. $\lambda_{\mathrm{exc}}=440 \mathrm{~nm}, \lambda_{\mathrm{em}}=650 \mathrm{~nm}$. The drawn line is the best least-squares fit to eq 7 .

be $10^{11}-10^{12} \mathrm{~s}^{-1}$. This leads to an estimate of the exciton diffusion length of $5-15 \mathrm{~nm}$, in agreement with the value obtained from the steady-state fluorescence spectra, indicating that the exciton diffusion length is not determined by the size of the aggregates, since then the value derived from steadystate fluorescence would have been significantly lower than the value derived from $k_{1}$. Monte Carlo simulations ${ }^{25}$ performed for the decay time measurements result in a more accurate value for the donor-donor transfer rate of $W=(1.0 \pm 0.2) \times 10^{12}$ $\mathrm{s}^{-1}$

Fluorescence Anisotropy Decay Measurements. To obtain more detailed information about the orientation of the porphyrin molecules on the substrate, we measured the fluorescence anisotropy decay of undoped ZnTOPP films, in addition to their fluorescence decay. The anisotropy decay shown in Figure 12 could be satisfactorily fitted to a sum of two exponentials, i.e.

$$
r(t)=\beta_{1} \exp \left(-t / \varphi_{1}\right)+\beta_{2} \exp \left(-t / \varphi_{2}\right)
$$

where $\beta_{1}=0.24 \pm 0.12, \varphi_{1}=14 \pm 3 \mathrm{ps}, \beta_{2}=0.053 \pm 0.003$, and $\varphi_{2}>18 \mathrm{~ns}$. The initial anisotropy $\beta_{1}+\beta_{2}=0.29$ is significantly higher than that of a monomer porphyrin in solution (typically 0.1) and confirms the ordering of the porphyrin molecules on the substrate, as reported in the preceding paper. For the proposed molecular ordering of aggregates consisting of linear porphyrin stacks with a slipped-deck-of-cards configuration with all porphyrin molecules perpendicular to the substrate and an angle of incidence of $65^{\circ}$ for the excitation beam, the calculated initial anisotropy value is 0.24 in good agreement with the observed value. The anisotropy decay must be due to energy transfer within the film. Since the $\varphi_{1}$ value is 

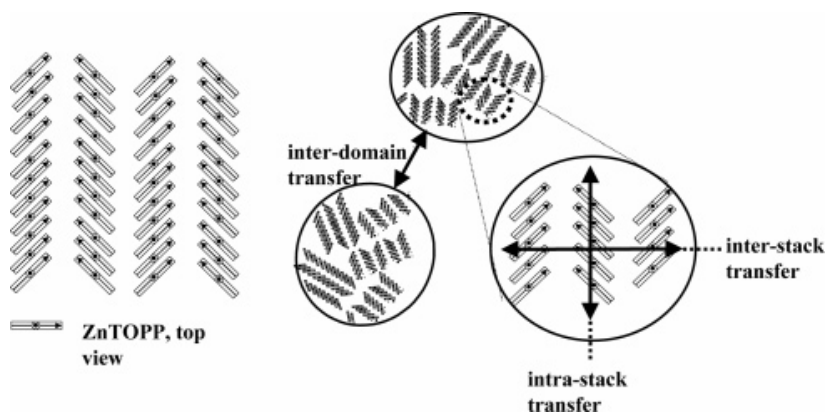

Figure 13. Proposed molecular ordering within a single domain of a spin-coated ZnTOPP film.

larger than that estimated for intrastack energy transfer, we ascribe it to interstack energy transfer. This assignment supports a parallel orientation of the porphyrin molecular planes within one stack, since orientations strongly deviating from a parallel one would result in a much faster anisotropy decay than observed. If interstack energy transfer were the only mechanism leading to depolarization, then the anisotropy decay is expected to be a single exponential with a constant value deviating from zero at long times after the excitation pulse. Although we could not determine an upper limit for $\varphi_{2}$, the experimental anisotropy decay probably is not a single exponential, indicating that more energy-transfer processes occur than those discussed above. Most likely the films consist of ordered domains, and the second component in the anisotropy decay could then be due to interdomain energy transfer.

The anisotropy decay due to interstack energy transfer implies that that the porphyrin stacks within one domain should at least have two different orientations. The observed values of the initial anisotropy and that of $\beta_{2}$ are in perfect agreement with a herringbone-type arrangement of porphyrin stacks within one domain as shown in Figure 13, with an angle of $90^{\circ}$ between the porphyrin planes in neighboring stacks, since for this arrangement values of 0.24 and 0.06 are calculated for the initial anisotropy and $\beta_{2}$, respectively. Note also that $\varphi_{1}^{-1}$ is not the probability for energy transfer from one ZnTOPP molecule in one stack to a particular ZnTOPP molecule in a neighboring stack but is the total probability for energy transfer from one stack as a whole to a neighboring one, constituting the summation of many ZnTOPP-ZnTOPP intermolecular energytransfer probabilities. Since the distance between two ZnTOPP molecules in one stack is relatively short compared to the distance between two stacks (see above), there are many molecules in a neighboring stack with a nonnegligible contribution to the total probability. The above-mentioned results are in quite good agreement with those from a Monte Carlo simulation of the kinetics of the excitation transport in these films. ${ }^{25}$

\section{Conclusions}

The main conclusions of this work are:

- In ZnTOPP films doped with $\mathrm{H}_{2}$ TOPP, the latter acts as a trap for excitation. In addition, thermally activated $\mathrm{H}_{2}$ TOPP to ZnTOPP back-transfer occurs. For CuTOPP, the quenching mechanism is basically different from that for $\mathrm{H}_{2} \mathrm{TOPP}$, resulting in completely irreversible excitation decay.

- The fluorescence of the films originates from the lowest excited singlet state that has a small excitonic interaction, resulting in almost localized exciton states. Therefore, excitation transport is diffusive rather than coherent. In accordance with this, the exciton diffusion length in these films is only $9 \pm 3$ $\mathrm{nm}$. This diffusion length is not determined by the stack length.
- From the results of fluorescence anisotropy decay, the intrastack energy transfer is shown to be quasi-one-dimensional with an estimated rate constant of $10^{11}-10^{12} \mathrm{~s}^{-1}$. The results suggest that additional fluorescence depolarization occurs through interstack energy transfer with a rate constant of $(7 \pm$ 2) $\times 10^{10} \mathrm{~s}^{-1}$ and possibly interdomain energy transfer.

- This work corroborates the main conclusions from the preceding paper, in particular the organization of the porphyrin molecules in the film with respect to the substrate.

Acknowledgment. This work was supported by The Netherlands Organization for Energy and Environment under Contract No. 146.100-024.4

\section{References and Notes}

(1) Energy Transfer Processes in Condensed Matter; DiBartolo, B. Ed.; Plenum Press: New York, 1983.

(2) Blasse, G. Prog. Solid State Chem. 1988, 18, 79

(3) Ueta, M.; Kanzaki, H.; Kobayashi, K.; Toyozawa, Y.; Hanamura E. Excitonic Processes in Solids; Springer-Verlag: Heidelberg, Germany, 1986.

(4) Singh J. Excitation Energy Transfer Processes in Condensed Matter Theory and Applications; Plenum Press: New York, 1994

(5) Blasse, G.; Dirksen, G. J.; Meijerink, A.; Van der Pol, J. F.; Neeleman, E.; Drenth, W. Chem. Phys. Lett. 1989, 154, 420.

(6) Markovitsi, D.; Tran-Thi, T.-H.; Briois, V.; Simon, J.; Ohta, K. J. Am. Chem. Soc. 1988, 110, 2001

(7) Markovitsi, D.; Germain, A.; Millié, P.; Lécuyer, P.; Gallos, L. K.; Argyrakis, P.; Bengs, H.; Ringsdorf, H. J. Phys. Chem. 1995, 99, 1005.

(8) Richter, B.; Kirstein, S. J. Chem. Phys. 1999, 111, 5191.

(9) Gómez, U.; Leonhardt, M.; Port, H.; Wolf, H. C. Chem. Phys. Lett. $1997,268,1$

(10) Kroese, J. E.; Koehorst, R. B. M.; Savenije, T. J. Adv. Funct. Mater. in press.

(11) Francis, A. H.; Kopelman, R. In Laser Spectroscopy of Solids; Yen, W. M., Selzer, P. M., Eds.; Topics in Applied Physics 49; SpringerVerlag: Berlin, 1981; Chapter 7.

(12) Ghiggino, K. P.; Smith, T. A. Prog. React. Kinet. 1993, 18, 375

(13) Powell, R. C.; Soos, Z. G. J. Lumin. 1975, 11, 1.

(14) Frolov, D.; Bagdonas, S.; Rotomskis, R. Lithuanian J. Phys. 2000, 40,228

(15) Gregg, B. A.; Sprague, J.; Peterson, M. W. J. Phys. Chem. B 1997, 101,5362

(16) Van Burgel, M.; Wiersma, D. A.; Duppen, K. J. Chem. Phys. 1995 102,20

(17) Kamalov, V. F.; Struganova, I.A.; Yoshihara, K. J. Phys. Chem. 1996, $100,8640$.

(18) De Boer, S.; Wiersma, D. A. Chem. Phys. Lett. 1990, 165, 45

(19) Gregg, B. A.; Fox, M. A.; Bard, A. J. J. Phys. Chem. 1989, 93, 4227

(20) Gregg, B. A.; Fox, M. A.; Bard, A. J. J. Phys. Chem. 1990, 94 1586.

(21) Gregg, B. A.; Kim, Y. I. J. Phys. Chem. 1994, 98, 2412

(22) Montroll, E. W. Proc. Symp. Appl. Math. Soc. 1964, 16, 193.

(23) Chandrasekhar, S. In Handbook of Liquid Crystals, Low Molecular Weight Liquid Crystals II; Demus, D., Goodby, J., Gray, G. W., Spiess, H.-W., Vill, V., Eds.; Wiley-VCH: Weinheim, Germany, 1998; Vol. 2B, Chapter 8

(24) Kerp, H. R.; Westerduin, K.; Van Veen, A.; Van Faassen, E. E. $J$. Mater. Res. 2001, 16, 503

(25) (a) Yatskou, M. M., Ph.D. Thesis, Wageningen University, 2001 (b) Yatskou, M. M.; Donker, H.; Novikov, E. G.; Koehorst, R. B. M.; van Hoek, A.; Apanasovich, V. V.; Schaafsma, T. J. J. Phys. Chem. A 2001 , 105, 9498. (c) Yatskou, M. M.; Donker, H.; Koehorst, R. B. M.; van Hoek, A.; Schaafsma, T. J. Chem. Phys. Lett. 2001, 345, 141. (d) Apanasovich, V. V.; Novikov, E. G.; Yatskou, M. M.; Koehorst, R. B. M.; Schaafsma, T. J.; van Hoek, A. J. Appl. Spectrosc. 1999, 66, 613.

(26) Gouterman, M. In The Porphyrins; Dolphin, D., Ed.; Academic Press: New York, 1978; Vol. III, Chapter 1.

(27) Adler, A. D.; Longo, F. R.; Kampas, F.; Kim, J. J. Inorg. Nucl. Chem. 1970, 32, 2443

(28) Adler, A. D.; Longo, F. R.; Shergalis, W. J. Am. Chem. Soc. 1964, 86,3145

(29) Little, R. G.; Anton, J. A.; Loach, P. A.; Ibers, J. A. J. Heterocycl. Chem. 1975, 12, 343

(30) van Hoek, A.; Visser, A. J. W. G. Rev. Sci. Instrum. 1981, 52 , 1199

(31) Vos, K.; van Hoek, A.; Visser, A. J. W. G. Eur. J. Biochem. 1987 165,55 
(32) van Hoek, A.; Visser, A. J. W. G. Anal. Instrum. 1985, 14, 359.

(33) Novikov, E. G.; van Hoek, A.; Visser, A. J. W. G.; Hofstraat, J. W. Opt. Commun. 1999, 166, 189.

(34) Humphrey-Baker, R.; Kalyanasundaram, K. J. Photochem. 1985, 31,105 .

(35) Nardo, J. V.; Dawson, J. H. Inorg. Chim. Acta 1986, 123, 9.

(36) Miller, J. R.; Dorough, G. D. J. Am. Chem. Soc. 1952, 74, 3977.

(37) Bonham, J. S.; Gouterman, M.; Howell, D. B. J. Lumin. 1975, 10 295.

(38) Kampas, F. J.; Gouterman, M. J. Lumin. 1976, 14, 121

(39) Kampas, F. J.; Gouterman, M. J. Phys. Chem. 1977, 81, 690

(40) Blondeel, G.; Harriman, A.; Porter, G.; Wilowska, A. J. Chem. Soc., Faraday Trans. 1984, 80, 867.

(41) Hofstra, U.; Koehorst, R. B. M.; Schaafsma, T. J. Chem. Phys. Lett. 1986, 130, 555.

(42) Brookfield, R. L.; Ellul, H.; Harriman, A. Faraday Trans. 1985 $2,1837$.

(43) Kroon, J. M.; Sudhölter, E. J. R.; Schenning, A. P. H. J.; Nolte, R. J. M. Langmuir 1995, 11, 214

(44) Kroon, J. M.; Koehorst, R. B. M.; van Dijk, M.; Sanders, G. M.; Sudhölter, E. J. R. J. Mater. Chem. 1997, 7, 615.
(45) Shimizu, Y.; Miya, M.; Nagata, A.; Ohta, K.; Yamamoto, I.; Kusabayashi, S. Liq. Cryst. 1993, 14, 795.

(46) Shimizu, Y.; Higashiyama, T.; Fuchita, T. Thin Solid Films 1998, $331,279$.

(47) Kerp, H. R.; Donker, H.; Koehorst, R. B. M.; Schaafsma, T. J.; Van Faassen, E. E. Chem. Phys. Lett. 1998, 298, 302.

(48) Movaghar, B.; Sauer, G. W.; Würtz, D. J. Stat. Phys. 1982, 27, 73.

(49) Dlott, D. D.; Fayer, M. D.; Wieting, R. D. J. Chem. Phys. 1977, 67,3808 .

(50) Wieting, R. D.; Fayer, M. D.; Dlott, D. D. J. Chem. Phys. 1978 69, 1996.

(51) Dlott, D. D.; Fayer, M. D.; Wieting, R. D. J. Chem. Phys. 1978, 69, 2752.

(52) Zumofen, G.; Blumen, A. Chem. Phys. Lett. 1982, 88, 63.

(53) Montroll, E. W. J. Math. Phys. 1969, 10, 3510

(54) Inokuti, M.; Hirayama, F. J. Chem. Phys. 1965, 43, 1978.

(55) Klafter, J.; Blumen, A. J. J. Chem. Phys. 1984, 80, 875.

(56) Klafter, J.; Blumen, A. J.; Zumofen, G. J. J. Chem. Phys. 1986, $84,1397$. 